Proc. of the 15th Int. Workshop on Slow Positron Beam Techniques and Applications, Prague, September 2-6, 2019

\title{
Positron Annihilation-Induced Auger Electron Spectroscopy Measurements of a $\mathrm{TiO}_{2}(110)$ Surface
}

\begin{abstract}
A.J. Fairchild*, V.A. Chirayath, R.W. Gladen, A.R. Koymen, and A.H. Weiss University of Texas at Arlington, Department of Physics Arlington, Texas 76019, USA

We report here positron annihilation-induced Auger electron spectroscopy measurements of an as received $\mathrm{TiO}_{2}(110)$ surface. We observe peaks at $\sim 250 \mathrm{eV}$ and $\sim 500 \mathrm{eV}$ due to the Auger decay of annihilation-induced $1 s$ holes in carbon $(\mathrm{C} \mathrm{KVV})$, and oxygen $(\mathrm{O} \mathrm{KVV})$ respectively. In addition to these KVV Auger peaks we observe a broad, low energy peak extending up to $15 \mathrm{eV}$. Since the incident positron beam energy of $1.5 \mathrm{eV}$ is well below the energy threshold required to directly knock out electrons with kinetic energies up to $15 \mathrm{eV}$, this peak must be Auger-related. Based on energy conservation requirements and its considerable spectral weight, we argue that this low energy peak is principally due to the Auger decay of both annihilation induced $2 s$ core holes in oxygen and annihilation induced valence holes in carbon.
\end{abstract}

DOI: 10.12693/APhysPolA.137.87

PACS/topics: 78.70.Bj, 41.75.Fr, 36.10.Dr

\section{Introduction}

Positron annihilation-induced Auger electron spectroscopy (PAES) is a non-destructive surface analysis technique with top-most atomic layer selectivity. The surface selectivity of PAES stems from the fact that the positron wave function in the surface state decays rapidly below the top-most atomic layer [1]. In PAES, positron beam energies well below the electron work function can be used to initiate the Auger process allowing for the complete elimination of the obscuring secondary electron background found in other similar techniques [2]. Experimental determination of the background-free, spectra of electrons emitted as a result of Auger transitions, down to $0 \mathrm{eV}$, is important for unravelling the complex, correlated Auger decay pathways of core and deep valence holes [3-7]. PAES studies of $\mathrm{TiO}_{2}(110)$ can also provide important insights relevant to the understanding of positron-stimulated desorption of $\mathrm{O}^{+}$from $\mathrm{TiO}_{2}(110)$. In particular, experimental demonstration of the positron surface state on the $\mathrm{TiO}_{2}(110)$ surface, measurement of the relative annihilation probabilities for the oxygen and titanium core levels and the experimental observation of multi-electron emission are all important for the current understanding of positron-stimulated desorption of $\mathrm{O}^{+}[8]$.

In this manuscript, preliminary (ToF)-PAES measurements of an as received $\mathrm{TiO}_{2}(110)$ surface with positron beam energies of $1.5 \mathrm{eV}$ and $47 \mathrm{eV}$ are presented. In the $1.5 \mathrm{eV}$ data we observe peaks corresponding to Auger decay of annihilation induced $1 s$ holes in carbon (C KVV at $250 \mathrm{eV}$ ) and oxygen (O KVV at $500 \mathrm{eV}$ ).

\footnotetext{
* corresponding author; e-mail:

alexander.fairchild@mavs . uta.edu
}

We additionally observe a broad, low energy peak extending up to $15 \mathrm{eV}$. We posit that some of the spectral weights in this peak are due to a C VVV Auger transition. In addition, our analysis suggests that a significant amount of the low energy peak is due to an O LVV transition. The core Auger process (O LVV) is initiated when a positron in the surface state annihilates with a $2 s$ electron in oxygen resulting in a less tightly bound valence electron filling this $2 s$ hole. The energy associated with this filling of the hole is then coupled to a third electron in the valence band which can escape into the vacuum as an Auger electron. The valence Auger process (C VVV) is similar except that the initial hole is in the valence band of carbon. This band is sufficiently deep to energetically support Auger electron emission [1]. Lastly, the system was configured to increase the energy separation between the $\mathrm{C}$ KVV and O KVV Auger peaks. We did not observe the Ti LMM (386 eV) or Ti LMV (410 eV) Auger peaks. The paper concludes with an analysis of the relative integrated intensities of the observed Auger peaks.

\section{Experimental setup}

The ToF-PAES spectrometer at the University of Texas at Arlington (UTA) has been described in detail elsewhere [2]. Briefly stated, positrons from a ${ }^{22} \mathrm{Na}$ source are first moderated using a thin tungsten foil in transmission geometry before being magnetically guided to the sample surface. The positron-induced electrons ejected from the sample have their momentum parallelized by a magnetic gradient near the sample. The ToF of the ejected electrons is measured as the time difference between the detection of the $511 \mathrm{keV}$ annihilation gamma rays by a fast scintillator and the detection of the electrons by a microchannel plate (MCP). Figure 1 is a schematic representation of the ToF-PAES spectrometer. The fast scintillator (2 inch NaI) is mounted $2 \mathrm{~cm}$ away from the sample. The sample, $99.99 \%$ rutile 


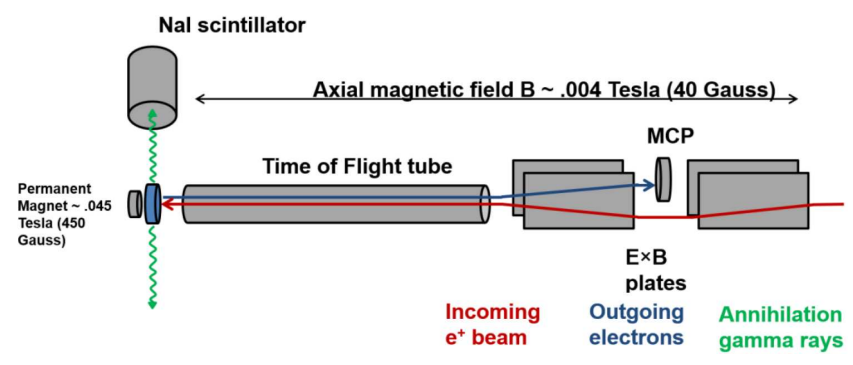

Fig. 1. Schematic representation of the TOF-PAES spectrometer at UTA. Positrons are magnetically guided to the sample by two sets of $\boldsymbol{E} \times \boldsymbol{B}$ plates. Positroninduced electrons have their momentum parallelized by a magnetic gradient near the sample. After traveling through a $1 \mathrm{~m}$ stainless steel tube biased at a constant potential, the positron-induced electrons are drifted by a pair of $\boldsymbol{E} \times \boldsymbol{B}$ plates onto the MCP. The kinetic energy of the electrons is measured using the ToF of the electrons, which is determined from the time difference between the detection of the annihilation gamma ray and the detection of the electron.

titanium(IV) oxide single crystal substrate (110), was purchased from Sigma-Aldrich. The sample was installed into the chamber without cleaning after prolonged storage in a plastic bag. The measurements were made without any further surface modifications to the sample after baking the chamber to approximately $375 \mathrm{~K}$ for $48 \mathrm{~h}$ to obtain a base pressure better than $10^{-8} \mathrm{~Pa}$.

\section{Results and Discussion}

Figure 2 is the ToF-PAES spectrum from $\mathrm{TiO}_{2}(110)$ measured using a positron beam energy of $1.5 \mathrm{eV}$. We observe two high energy (low ToF) peaks corresponding to electron emission from the Auger decay of annihilationinduced $1 s$ holes in carbon (C KVV $250 \mathrm{eV}$ ) and oxygen $(\mathrm{O} K V V 500 \mathrm{eV})$. These peak energies are consistent with previous measurements of $\mathrm{C} \mathrm{KVV}(263 \mathrm{eV})$ and $\mathrm{O}$ KVV (503 eV) [9]. We note that the peak energies measured with our spectrometer are lower due to the incomplete parallelization of the outgoing Auger electron momentum [10]. In addition, we observe a broad low energy (high ToF) peak. The line shape of this low energy peak in energy space is shown in Fig. 3. It has peak intensity at $2 \mathrm{eV}$ and extends up to $15 \mathrm{eV}$ with an integrated intensity 107 times the integrated C KVV intensity and 61 times the integrated $\mathrm{O} \mathrm{KVV}$ intensity. The integrated intensities quoted have been corrected for the transport efficiency through our ToF-PAES spectrometer using a model spectrometer detailed in [11]. The peak is ascribed to two positron-induced Auger processes: O LVV Auger processes initiated by annihilationinduced $2 s$ holes in oxygen and $\mathrm{C}$ VVV Auger processes initiated by annihilation-induced valence holes in carbon. Photoemission measurements of $\mathrm{TiO}_{2}(110)$ have shown a valence band width of $6 \mathrm{eV}$ and $\mathrm{O} 2 s$ levels as deep

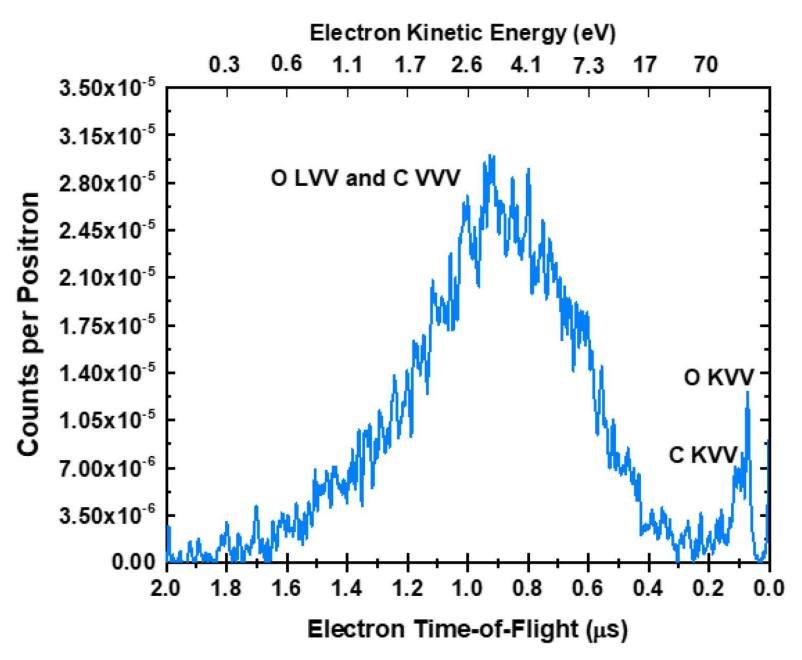

Fig. 2. ToF-PAES from $\mathrm{TiO}_{2}(110)$ measured using an incident positron beam energy of $1.5 \mathrm{eV}$. Auger peaks associated with C KVV $(250 \mathrm{eV})$ and O KVV (500 eV) Auger transitions are present alongside a broad low energy peak which is ascribed to combined O LVV and $\mathrm{C}$ VVV Auger processes. The spectrum has been normalized to the number of background subtracted NaI counts.

TABLE I

PAES integrated intensities obtained for each of the observed Auger peaks in Fig. 2. The intensities have been corrected using estimates of the spectrometer transport efficiencies but not for the detector efficiencies.

\begin{tabular}{l|c|c|c}
\hline \hline & O LVV $/ \mathrm{C} \mathrm{VVV}$ & $\mathrm{C} \mathrm{KVV}$ & $\mathrm{O} \mathrm{KVV}$ \\
\hline $\begin{array}{l}\text { integrated } \\
\text { intensity }\end{array}$ & $9.4 \times 10^{-3}$ & $8.8 \times 10^{-5}$ & $1.5 \times 10^{-4}$ \\
\hline $\begin{array}{l}\text { relative } \\
\text { intensity }\end{array}$ & 61 & 0.6 & 1
\end{tabular}

as $25 \mathrm{eV}[12,13]$. Therefore, it is energetically possible for an LVV Auger decay of $\mathrm{O} 2 s$ holes with binding energies up to $25 \mathrm{eV}$ to produce Auger electrons with kinetic energies up to $15 \mathrm{eV}$. Additionally, the narrow valence band width makes VVV Auger processes from $\mathrm{TiO}_{2}$ energetically forbidden. The presence of carbon on the as received sample does mean that some $\mathrm{C} \mathrm{VVV}$ Auger emission is expected, but our previous measurements of single-layer graphene have found that the integrated C VVV Auger intensity is only about a factor of 20 times the integrated C KVV Auger intensity, not over 100 times as seen here [1]. Furthermore, a low energy electron emission mechanism associated with the decay of $\mathrm{O} 1 s$ annihilation-induced holes could at best explain a relative integrated intensity of 2 if every O KVV Auger electron was accompanied by a low energy electron.

The ratio of PAES integrated intensities are listed in Table I and provides a first estimate of the relative positron annihilation probabilities for the different core and valence levels. From the ratios of the low 


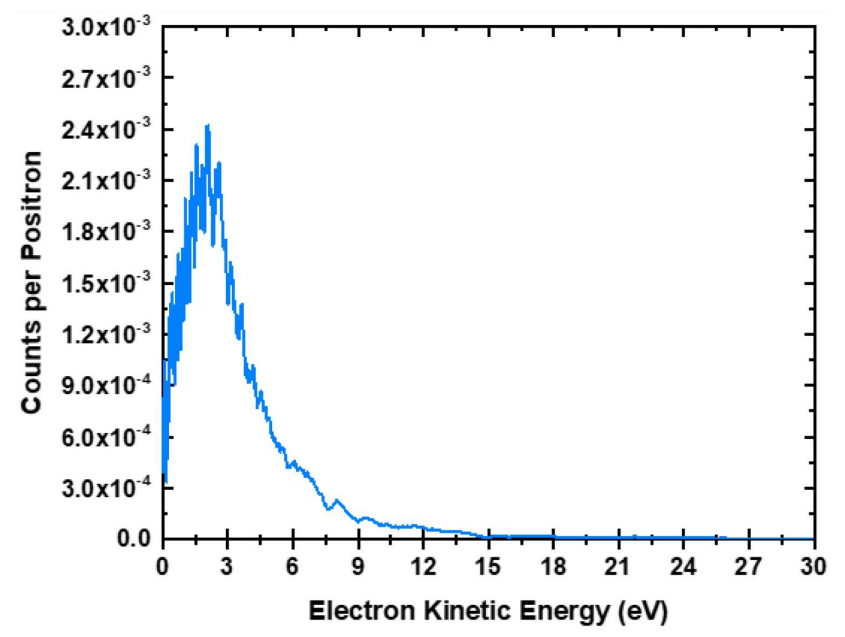

Fig. 3. Energy converted ToF-PAES of Fig. 2 plotted up to $30 \mathrm{eV}$. The low energy peak has a maximum at $2 \mathrm{eV}$, extends up to $15 \mathrm{eV}$ and has an integrated intensity 61 times that of the O KVV Auger peak and 107 times that of the C KVV integrated intensity. The spectrum has been normalized to the number of background subtracted NaI counts.

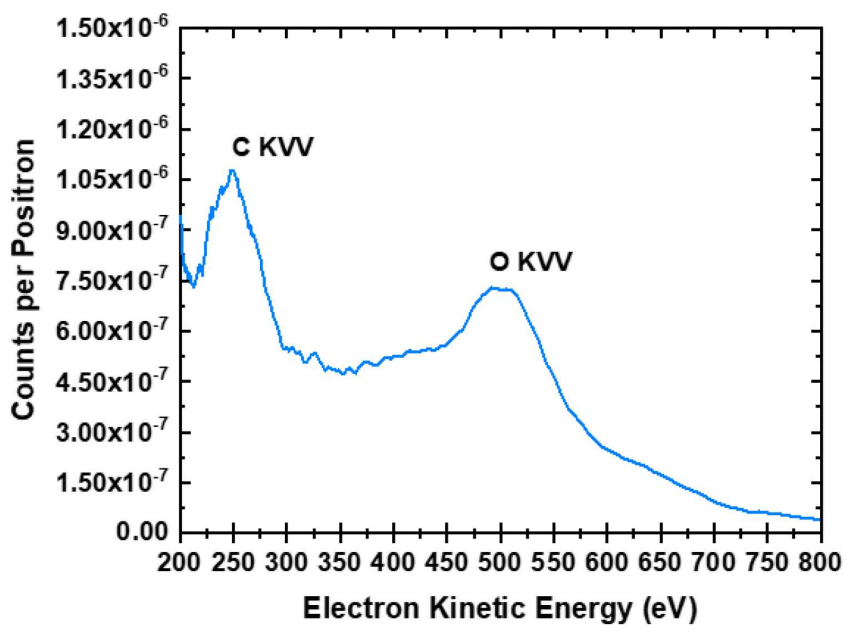

Fig. 4. Energy converted ToF-PAES for a positron beam energy of $47 \mathrm{eV}$. The beam optics were set to obtain increased energy separation between the C KVV and $\mathrm{O} \mathrm{KVV}$ Auger peaks. We do not observe the Ti LMM $(386 \mathrm{eV})$ or LMV $(410 \mathrm{eV})$ Auger peaks. The spectrum has been normalized to the number of background subtracted NaI counts.

energy peak to the two KVV peaks we can conclude that positrons are an order of magnitude more likely to annihilate with the oxygen $2 s$ or carbon valence levels than either the oxygen $1 s$ or carbon $1 s$ levels for the as received $\mathrm{TiO}_{2}(110)$ sample. Finally, since the incident positron beam energy of $1.5 \mathrm{eV}$ is well below the energy threshold required to excite secondary electron processes, the signals we are observing are entirely due to the Auger decay of annihilation-induced holes.
Lastly, it is worth considering the noticeable absence of any titanium derived Auger signals in our measurements. In Fig. 3 we do not see the Ti MVV Auger peak $(18 \mathrm{eV})$, which has previously been observed for $\mathrm{TiO}_{2}(110)$ in Auger-photoelectron coincidence spectroscopy (APECS) [14]. In Fig. 2, however, the presence or absence of the Ti LMM $(386 \mathrm{eV})$ or Ti LMV $(410 \mathrm{eV})$ is more difficult to determine. Therefore, we have carried out additional ToF-PAES measurements using a positron beam energy of $47 \mathrm{eV}$, a ToF tube bias of $-120 \mathrm{~V}$ and adjusted the system's optics to give increased energy separation between the C KVV and O KVV peaks. The energy converted spectrum for these measurements is show in Fig. 4. With the increased energy separation between the peaks we do not see either of the titanium derived Auger peaks. We believe the lack of Ti derived Auger signals is due to the reduced positron wave function overlap with titanium due to the presence of oxygen and carbon on the surface.

\section{Conclusions}

We have presented ToF-PAES measurements of an as received $\mathrm{TiO}_{2}(110)$ surface for two incident positron beam energies, $1.5 \mathrm{eV}$ and $47 \mathrm{eV}$. These measurements provide direct experimental proof for a positron surface state on $\mathrm{TiO}_{2}(110)$. We observe peaks from the Auger decay of annihilation-induced holes in the $1 \mathrm{~s}$ levels of oxygen $(\mathrm{O} \mathrm{KVV})$ and carbon $(\mathrm{C} \mathrm{KVV})$ in addition to a low energy Auger peak due to the Auger decay of both annihilation-induced holes in the $2 s$ levels of oxygen (O LVV) and valence levels of carbon (C VVV). Notably, we do not observe any peaks due to the Auger decay of annihilation-induced $\mathrm{Ti} 2 p$ or $3 p$ holes. Future work will focus on treating the surface to remove any carbon contamination and calculating more precisely the relative annihilation probabilities for the clean, stoichiometric surface.

\section{Acknowledgments}

This work was supported by NSF grants DMR 1508719 \& DMR 1338130 and Welch Foundation grant No. Y-1968-20180324.

\section{References}

[1] V.A. Chirayath, V. Callewaert, A.J. Fairchild, M.D. Chrysler, R.W. Gladen, A.D. Mcdonald, S.K. Imam, K. Shastry, A.R. Koymen, R. Saniz, B. Barbiellini, K. Rajeshwar, B. Partoens, A.H. Weiss, Nat. Commun. 8, 16116 (2017).

[2] S. Mukherjee, K. Shastry, C.V. Anto, P.V. Joglekar, M.P. Nadesalingam, S. Xie, N. Jiang, A.H. Weiss, Rev. Sci. Instrum. 87, 035114 (2016).

[3] S.F. Mukherjee, K. Shastry, A.H. Weiss, Phys. Rev. B 84, 155109 (2011).

[4] E. Jensen, R.A. Bartynski, R.F. Garrett, S.L. Hulbert, E.D. Johnson, C.C. Kao, Phys. Rev. B 45, 13636 (1992). 
[5] S. Satyal, P.V. Joglekar, K. Shastry, S. Kalaskar, Q. Dong, S.L. Hulbert, R.A. Bartynski, A.H. Weiss, J. Electron Spectrosc. 195, 66 (2014).

[6] Z. Wei, F.O. Schumann, C.H. Li, L. Behnke, G. Di Filippo, G. Stefani, J. Kirschner, Phys. Rev. Lett. 113, 267603 (2014).

[7] P.V. Joglekar, R.W. Gladen, V.A. Chirayath, A.J. Fairchild, S. Kalaskar, K. Shastry, Q. Dong, S.L. Hulbert, R.A. Bartynski, W.S.M. Werner, A.H. Weiss, J. Electron Spectrosc. 235, 16 (2019).

[8] T. Tachibana, T. Yamashita, M. Nagira, H. Yabuki, Y. Nagashima, Sci. Rep. 8, 7197 (2018).

[9] T. Sekine, Y. Nagasawa, M. Kudoh, Y. Sakai, A.S. Parkes, J.D. Geller, A. Mogami, K. Hirata, in: Handbook of Auger Electron Spectroscopy, JEOL LTD., Akishima, Tokyo 1982.
[10] C. Lei, D. Mehl, A.R. Koymen, F. Gotwald, M. Jibaly, A. Weiss, Rev. Sci. Instrum. 60, (1989).

[11] A.J. Fairchild, V.A. Chirayath, R.W. Gladen, M.D. Chrysler, A.R. Koymen, A.H. Weiss, J. Phys. Conf. Ser 791, 012030 (2017).

[12] R. Heise, R. Courths, Surf. Sci. 110, 658 (1993).

[13] S. Sampath, P. Maydannik, T. Ivanova, M. Shestakova, T. Homola, A. Bryukvin, M. Sillanpaa, R. Nagumothu, V. Alagan, Superlattice Microst. 97, 155 (2016).

[14] W.K. Siu, R.A. Bartynski, S.L. Hulbert, J. Chem. Phys. 113, 10697 (2000). 